\title{
Lidil
}

Revue de linguistique et de didactique des langues

$54 \mid 2016$

La phrase en production d'écrits, approches nouvelles en didactique

\section{La phrase dans le texte. L'exemple de phrases de dialogue dans un corpus d'école élémentaire}

The Sentence in the Text: The Example of Dialogue Sentences in an Elementary

School Corpus

Catherine Boré et Catherine Bosredon

(2) OpenEdition

Journals

Édition électronique

URL : http://journals.openedition.org/lidil/4073

DOI : $10.4000 /$ lidil.4073

ISSN : 1960-6052

Éditeur

UGA Éditions/Université Grenoble Alpes

Édition imprimée

Date de publication : 20 novembre 2016

Pagination : 115-133

ISBN : 978-2-84310-339-1

ISSN : $1146-6480$

Référence électronique

Catherine Boré et Catherine Bosredon, « La phrase dans le texte. L'exemple de phrases de dialogue dans un corpus d'école élémentaire », Lidil [En ligne], 54 | 2016, mis en ligne le 01 janvier 2017, consulté le 29 octobre 2020. URL : http://journals.openedition.org/lidil/4073 ; DOI : https://doi.org/ 10.4000/lidil.4073 


\title{
La phrase dans le texte. L'exemple de phrases de dialogue dans un corpus d'école élémentaire
}

\author{
Catherine Boré et Catherine Bosredon*
}

\begin{abstract}
RÉSUMÉ
L'article aborde la question des rapports entre la phrase et le texte, en partant d'un couple de phrases de dialogue conçues comme une unité textuelle porteuse de deux énoncés distincts. Il s'appuie sur un corpus de productions écrites d'élèves de 8-11 ans issus de six classes différentes qui ont reçu la consigne identique d'insérer ces phrases dans leur texte au cours de deux temps d'écriture et réécriture. Les résultats font apparaitre une rupture au niveau de la dernière classe d'école élémentaire dans la prise en compte des contraintes énonciatives, syntaxiques et sémantiques réciproques de la phrase et du texte. Sur le plan didactique, cette étude empirique montre peu de changements dans la réécriture, sauf lorsque l'enseignant rend explicites les inférences contenues dans le dialogue.
\end{abstract}

\begin{abstract}
This article deals on the relationship between the sentence and the text, starting from two dialogue sentences which are defined as a textual unit carrying two distinct utterances. The study is based on a corpus of written narratives by pupils aged 8-11 coming from six different classes. They all received the same instruction to insert the couple of sentences in their text, during two separate times. Considering the results, a gap appears at the last elementary school level, from the time when pupils can deal with many constraints (enunciative, syntaxic and semantic) both between the sentence and the text. Didactically, this empirical analysis shows little change when pupils rewrite their text, unless the teacher makes explicit the inferences contained in the dialogue.
\end{abstract}

* Université de Cergy-Pontoise, laboratoire EMA - EA 4507, ESPE. 


\section{Introduction}

L'analyse de Benveniste (1966) selon laquelle, en passant du mot à la phrase, on change «d'univers», opposait le sémiotique (combinaison contrainte de signes) au sémantique, dont la phrase serait le premier maillon. La phrase dès lors se trouvait prise dans un rapport dialectique selon le point de vue adopté : l'appréhender hors de son milieu naturel qui est le discours conduirait à s'intéresser à l'organisation de ses constituants et à la dépendance des unités qui la composent. Mais dès l'instant où la phrase est construite, elle forme texte et sens, recevant et produisant d'autres contraintes. F. Rastier, qui a pris la sémantique pour objet, n'a cessé de signaler l'influence du global sur le local (2005), tandis que D. Legallois et F. Neveu posaient les rapports de la phrase et du texte en ces termes lors de leur présentation des journées d'étude du Crisco des 3 et 4 décembre 2009 : «Quel que soit le format choisi - phrase, période, proposition - chacune de ces unités, considérée dans son élément naturel, à savoir le texte, fait l'objet de déterminations qui peuvent être d'ordres différents.»

Parmi celles-ci, se trouve le fait que la phrase réponde à un problème ou une question préalable dans le texte, ce qui contraint son organisation, et inversement, «dans un mouvement circulaire, la phrase, objet de détermination textuelle, est également anticipatrice du texte ${ }^{1} \gg$.

Autrement dit, la syntaxe est-elle au service du texte ou le texte informe-t-il la syntaxe?

En examinant un corpus de productions écrites de l'école élémentaire (cycle 3) répondant à une consigne d'insertion de phrases de dialogue dans leur production, nous revenons sur cette question sous un angle particulier et limité : nous nous demanderons comment les problèmes énonciatifs, syntaxiques et sémantiques posés par l'insertion de ces phrases dans leur texte ont pu être perçus et traités par ces scripteurs.

1. Atelier de linguistique du Crisco, Ce que le texte fait à la phrase, Journées d'étude des 3 et 4 novembre 2009. Disponible sur <www.crisco.unicaen.fr/ IMG/pdf/ce_que_le_texte_fait_a_la_phrasederniereversion.pdf $>$. 


\section{Corpus, objectif de l'étude, méthodologie}

Le corpus a été réuni dans le cadre d'une recherche doctorale ${ }^{2}$ en cours. Seule une partie est présentée ici. La partie retenue se compose des productions écrites de six classes issues de trois établissements différents de Paris et de la région parisienne, de niveau socio-culturel comparable correspondant aux classes moyennes avec une petite minorité d'élèves en difficulté par classe. Sont représentées 2 classes de CE2, 2 classes de CM1, 2 classes de $\mathrm{CM}_{2}{ }^{3}$. Les enseignants ont accepté de se soumettre au protocole de production d'écrit, similaire dans les trois niveaux, et ont bénéficié de l'accompagnement de la doctorante à l'origine de la demande.

Six vignettes extraites de l'album Le gardien de l'oubli ${ }^{4}$ sont proposées aux élèves, à partir desquelles ils devront écrire une histoire fictive. Lors de la $1^{\text {re }}$ séance orale, ils repèrent des indices dans les images pour construire une trame. Sous l'une des images se trouvent deux phrases ${ }^{5}$ de dialogue, extraites de l'album, qu'ils auront à insérer dans leur texte :

- Tu as perdu quelque chose? lui demanda Anne-Lise.

- Ma toupie chinoise sur le chemin de l'école.

Une seconde séance d'écriture intervient huit jours plus tard, à partir des copies annotées accompagnées de consignes de relecture, et, pour une classe de CM2, d'une grille d'aide à la réécriture.

Le tableau 1 récapitule la répartition des 283 copies obtenues, par niveau, par établissement et par version.

2. Merci à Catherine Bosredon d'avoir accepté que soit utilisée une partie de son corpus pour cet article.

3. Le recueil a été fait en 2013, dans la logique de ce qui était le cycle 3, et ne peut donc, de ce fait, tenir compte du nouveau découpage en 4 cycles de la rentrée de septembre 2015.

4. J.-M. Gisbert \& A. Ruano (1991), Le gardien de l'oubli (L. Merle d'Aubigné, trad.), Paris : Syros jeunesse. L'ouvrage se trouve dans les listes de lectures recommandées par le ministère de l'Éducation.

5. Ce découpage sera discuté plus loin. 


\begin{tabular}{|c|c|c|c|c|}
\hline CLASSES & $\begin{array}{l}\text { Nombre } \\
\text { d'élèves }\end{array}$ & $\begin{array}{c}\text { Nombre } \\
\text { d'élèves } \\
\text { présents V1 }\end{array}$ & $\begin{array}{c}\text { Nombre } \\
\text { d'élèves } \\
\text { présents V2 }\end{array}$ & $\begin{array}{l}\text { Nombre de } \\
\text { copies }\end{array}$ \\
\hline CE2 PA & 25 & 23 & 24 & \\
\hline CE2 JO & 28 & 28 & 27 & \\
\hline TOTAL CE2 & 53 & 51 & 51 & 102 \\
\hline CM1 Pyr A & 24 & 21 & 24 & \\
\hline CM2 Pyr B & 20 & 18 & 19 & \\
\hline TOTAL CM1 & 44 & 39 & 43 & 82 \\
\hline $\mathrm{CM} 2 \mathrm{JO}$ & 30 & 28 & 30 & \\
\hline CM2 Pyr C & 22 & 21 & 20 & \\
\hline TOTAL CM2 & 52 & 49 & 50 & 99 \\
\hline $\begin{array}{l}\text { TOTAL } \\
\text { GLOBAL }\end{array}$ & 149 & 139 & 144 & 283 \\
\hline
\end{tabular}

Tableau 1. - Répartition du corpus.

L'objectif du présent article n'est pas directement didactique, en ce sens qu'il ne s'agit pas de tester la capacité des élèves à mieux insérer ces phrases dans leur texte entre les deux versions grâce au travail fait en classe avec l'enseignant.

Notre objectif se situe en amont. Nous analysons les difficultés que rencontrent des élèves «tout venant» du cycle 3 dans une tâche scolaire ordinaire, mais dont la réalisation est plus complexe que ne le pensent généralement les enseignants. En effet, il n'existe pas de phrase standard SVO, qu'il serait énonciativement «neutre» d'insérer : celles qui sont proposées dans ce travail de production d'écrit, nous le verrons, sont porteuses de formes attestant de leur origine énonciative, et ceci implique, pour l'élève, de reconstituer le contexte sémantique et syntaxique ayant amené leur production. À l'inverse, le texte créé par l'élève développe ses contraintes propres qui viennent buter sur les phrases proposées au point de les modifier. Nous montrerons que ce double mouvement, qui existe dans toute production d'écrit, est représenté aux différents niveaux du cycle et que c'est la méconnaissance, chez les enseignants, des difficultés des élèves à tenir compte de ces doubles contraintes qui rend souvent inefficace la réécriture en deux versions.

Sur le plan méthodologique, nous avons adopté une méthode non expérimentale, dans la mesure où les élèves écrivent dans leur contexte didactique habituel avec leurs enseignants; mais le caractère identique 
de l'exercice et de son dispositif, aux trois niveaux du cycle, le nombre comparable de copies recueillies par niveau, la similitude des postures didactiques des enseignants, autorisent néanmoins le recueil d'éléments quantitatifs permettant de comparer les résultats obtenus au cycle scolaire observé.

\section{Dialogue, discours direct : unité textuelle à deux énoncés}

Plane (2006) remarquait que si le texte possède des propriétés intrinsèques comme «la cohésion, qui fait que le sens transite au-delà des segments qui le portent, la topicalité, liée au fait que le texte se donne un objet et qu'il le construit», celles-ci «ne fournissent pas les éléments susceptibles de rendre compte de façon satisfaisante de certains textes dialogaux, procédant du tissage de deux énoncés» (p. 41). En effet, la (les) phrase(s) d'insertion proposée(s) aux élèves sont des phrases de discours rapporté direct qui forment un système interrogatif composé d'une question (P1) introduite par une incise, et d'une réponse (P2) sous forme elliptique ou averbale. Elles diffèrent du modèle de la phrase canonique enseignée à l'école qui est le plus souvent isolée, assertive, et verbale, la phrase averbale ou elliptique étant présentée comme exceptionnelle. Notons que ce sont des phrases authentiques de l'écrit, extraites du texte lu, et non construites artificiellement pour une expérience.

\subsection{La question ( $\left.P_{1}\right)$}

Sur le plan pragmatique, notons tout d'abord que l'interrogative est ambigüe : sur la forme, elle semble correspondre à une interrogation totale en oui/non, alors que la réponse «Ma toupie...» montre qu'il s'agit en fait d'une question portant sur une partie du prédicat dont l'interrogation correspondante pourrait être : Qu'as-tu perdu? ou Qu'estce que tu as perdu?

Par ailleurs, en l'absence d'inversion du sujet, l'interrogation n'est marquée que par la présence du point d'interrogation. Enfin, sur le plan énonciatif qui est l'angle que nous privilégions, cette interrogation est un discours direct, une mention de discours direct en l'occurrence, signalée par une incise parfois appelée «incise de citation». 


\subsection{L'incise}

Nous avons inclus l'incise dans la structure interrogative qu'elle est censée régir. Sur ce point, nous suivons Danlos et coll. (2010) : «La citation et l'incise de citation forment un agrégat soudé dans la mesure où une incise de citation demande la présence d'une citation.» (p. 2237) Cependant, pour ces auteurs, «il n'existe pas de lien argumental syntaxique entre le verbe de citation et la citation» (ibid.), mais une relation sémantico-discursive.

En dépit de la faiblesse rectionnelle du verbe de l'incise, celle-ci introduit bien un discours rapporté, autonymisé (Authier-Revuz, 1995), à la différence du dialogue qui alterne les répliques sans l'intervention d'un locuteur rapporteur ( $L$ dans le vocabulaire d'Authier).

Si l'on se place sur le plan textuel, l'incise appartient bien à la strate narrative du texte et, sur le plan énonciatif, l'incise manifeste à une place singulière la présence de L : elle signale (par le verbe) la représentation que se fait $\mathrm{L}$ du discours d'autrui.

Le fonctionnement de l'incise inclut donc obligatoirement la nomination de L sous une forme (identité lexicale) ou sous une autre (substitut pronominal). Dans notre exemple, l'incise introduit un discours (question) qui s'adresse à un allocutaire ${ }^{6}$. La réponse de l'allocutaire de P2 (Gabriel) est elle aussi rapportée, mais sans l'incise, ce qui crée une dissymétrie rendant difficile pour les jeunes scripteurs la compréhension du rôle exact de l'incise, comme nous le verrons dans l'analyse.

\subsection{La réponse $\left(P_{2}\right)$}

La réponse est une phrase elliptique selon Riegel et coll. (1994, p. 133). Mais il subsiste une portion prédicative ${ }^{7}$ «Ma toupie chinoise», qui, selon cette analyse, serait le complément du noyau verbal ellipsé «J'ai perdu».

Pourtant d'autres analyses sont possibles : Lefeuvre (2013) examinant un corpus oral de questions-réponses, considère le segment ellipsé comme une phrase averbale autonome, à la différence de Lacheret et

6. Bien entendu, il existe des incises limitées à un seul locuteur.

7. En l'occurrence, l'allocutaire répond au locuteur comme s'il lui avait posé une question partielle, et non pas totale. Nous verrons, dans l'analyse des résultats, que cela a troublé les scripteurs. 
coll. ${ }^{8}$ (cités par Lefeuvre) qui estiment que l'on peut considérer ce type de système comme «une seule unité rectionnelle gouvernée par un verbe» (ici «as perdu»). Le segment elliptique P2 (ici : «Ma toupie chinoise...») serait ainsi «dans la continuation du verbe de la première phrase, avec un "entassement" des compléments d'objet direct» («quelque chose» «toupie chinoise» dans notre cas) mais il y aurait bien deux actes illocutoires distincts (interrogation et assertion).

Selon cette dernière analyse, le système interrogatif à insérer est donc complexe et fortement hiérarchisé, l'incise régissant P1, qui régit P2, l'ensemble formant une unité.

Si nous nous sommes attardées sur l'argumentation de ces auteurs, c'est qu'un nombre non négligeable de scripteurs ${ }^{9}$ confrontés au problème d'insérer ces phrases dans leur texte, ont été amenés à les modifier, mettant par là même en évidence les conflits dans l'analyse syntaxique énonciative et sémantique dont elles sont porteuses. En effet, les modifications portent :

- sur le contexte gauche de la phrase interrogative au discours direct et, plus encore, sur son contexte droit, qui est l'incise;

- sur la forme de la phrase averbale : soit transformée en phrase verbale canonique, soit «saturée» par un modalisateur (en l'occurrence la prophrase oui) et développée ultérieurement par un ajout d'informations.

On peut dire alors que c'est la prise en compte de l'environnement textuel qui oblige les scripteurs à opérer des modifications sur les phrases à insérer. Mais inversement, celles-ci sont porteuses d'informations qui contraignent le texte à produire. Nous allons donc examiner ce double mouvement.

\section{La phrase contraint le texte : les modifications sémantiques}

Les élèves de chaque classe ont reçu avant la rédaction de leur texte (V1) des indications leur permettant de prêter attention aux difficultés

8. On pourrait aussi citer Blanche-Benveniste qui considère l'unicité du dialogue comme un tout sans distinguer les allocutaires (référence citée par Kuyumkuyan, 2013).

9. Nous donnons les chiffres et proportions dans l'analyse qui va suivre. 
sémantiques prévisibles. Cependant très vite se posent des difficultés liées aux inférences.

\subsection{Les problèmes liés aux inférences}

P1 n'est pas assertive; elle a la forme d'une interrogation totale qui réclame une confirmation : un «oui», implicite dans la réponse P2. Cette interrogation est déclenchée par un locuteur nommé dans l'incise dont le lecteur présuppose qu'il connait le personnage principal ou qu'il a des informations à lui communiquer. Néanmoins, si ce locuteur n'a pas été présenté antérieurement, rien n'empêche pour autant le lecteur de faire des présuppositions d'existence à partir du contexte (si l'école est nommée antérieurement, elle présuppose des camarades qui peuvent être locuteurs). Sur ce point (connaissance des interlocuteurs), on peut se fonder sur les règles de Lewis citées par Deloor (2012, p. 10) :

$\mathrm{La}$ «règle de compromis» (rule of accomodation) proposée par $\mathrm{D}$. Lewis (1979) formalise ce mécanisme basé sur la bonne volonté de l'interlocuteur :

If at time $T$ something is said that requires presupposition $P$ to be acceptable, and if $P$ is not presupposed just before, then $[\ldots]$ presupposition P comes into existence at T. (Lewis, $1979: 340$ ).

Mais P1 présuppose aussi que son locuteur pense que quelque chose a été perdu par le personnage principal. Certes, l'information que la toupie magique a été perdue est indispensable pour le lecteur. Elle est d'ailleurs notée par la quasi-totalité des scripteurs, quel que soit leur niveau. Mais cela ne suffit pas : comment en effet le locuteur de P1 peut-il poser sa question sans une information explicite dans le cotexte permettant de lui attribuer cette pensée ? C'est la principale difficulté sémantique.

On a donc cherché à savoir s'il y avait dans le cotexte antérieur ou postérieur à $\mathrm{P} 1$ et $\mathrm{P} 2$ des éléments référentiels permettant ou non d'identifier les locuteurs et des éléments justifiant la question du locuteur de P1.

\subsection{Analyse}

La méta-règle de répétition (Charolles, 1978) donne au texte son «fil conducteur» grâce à la reprise des personnages mais aussi des éléments se rapportant à la péripétie. 
67 élèves parmi ceux présents aux deux versions - soit la moitié ont caractérisé le personnage principal comme «un petit garçon» de 6 à 10 ans présenté dès la première phrase. Certains ont utilisé leurs connaissances du monde, le mettant en situation, lui prêtant des activités enfantines courantes qui engendreront la perte de la toupie; quelquesuns inventent de nouveaux personnages : une famille, des copains. $\mathrm{Si}$ les élèves de CE2 et de CM1 caractérisent de façon succincte le personnage de Gabriel ${ }^{10}$, la lecture montre qu'ils le mettent en scène en tant que personnage principal dont on connait l'identité. La chaine anaphorique est en général construite sur le modèle suivant : un GN (un petit garçon) suivi d'un GN expansé permettant de l'associer au référent (Gabriel) auquel succède dans la phrase suivante, le pronom personnel sujet $i l$ repris dans la P1 par le pronom personnel complément lui. Tous élaborent au moins : Gabriel $\rightarrow$ il $\rightarrow$ lui.

La majorité (67 élèves) caractérise uniquement le personnage principal. Toutefois, un tiers de ces jeunes scripteurs donne des éléments permettant de caractériser le second personnage nommé Anne-Lise dans l'incise, et permettant de l'identifier comme locutrice de la P2. Celle-ci est caractérisée comme amie, copine, fille, voisine, sœur par des élèves qui mettent en scène l'image 2 avant d'introduire le dialogue. Un palier est observable en CM2 puisque le nombre d'élèves qui établit ce lien double entre le CM1 et le CM2. Au total, seuls 19 élèves, tous niveaux confondus, ne donnent dans la V1 aucun élément permettant de caractériser les deux personnages.

Les procédures de répétition apparaissent également dans ce que Charolles nomme les recouvrements présuppositionnels ou dans les reprises d'inférence. On constate sur ce point des difficultés importantes. Certains plaquent les phrases à insérer sans aucun lien avec le reste : ce sont pour la plupart les scripteurs de CE2. La cohérence est rompue si les scripteurs n'apportent aucun autre élément nouveau qui permette au locuteur de poser sa question enfreignant ainsi la règle de progression (Charolles). La majorité des élèves (90) et la quasi-totalité des élèves de CE2 se heurtent à ce problème. C'est le cas du texte suivant (redondance et circularité) :

10. Le nom du personnage est donné au cours de la présentation des images de l'album. 
(1)

Gabriel a perdu sa toupie sur le chemin de l'école. Arrivé à l'école il retrouve/ Anne-Lise : «- tu as perdu quelque chose? demanda AnneLise./ - Ma toupie chinoise sur le chemin de l'école.» (Jus CE2 JO V1) ${ }^{11}$

Seuls 5 élèves de CE2, 12 de CM1 et 25 de CM2 insèrent la P1 en faisant en sorte que cette question puisse être posée de façon partiellement ou totalement pertinente et acceptable Ils évoquent certes la perte ou créent une situation au cours de laquelle la toupie disparait. Les verbes perdre, ne pas trouver, n'y était plus, figurent conjugués dans la phrase de récit précédant les phrases à insérer et sont repris par tu as perdu de la question posée (P1). Le champ lexical de la perte est complété par les verbes chercher, fouiller ou le nom recherche, montrant ainsi les actions qui vont se succéder et qui construisent la cohérence sémantique.

Plus d'un quart des élèves (tous niveaux, toutes versions) s'intéresse aux états des personnages et à leurs intentions: Gabriel est triste, malheureux ou pleure, n'est pas bien, boude, est bouleversé. Toutefois ces réussites sont partielles, car les scripteurs n'arrivent pas toujours à établir le lien textuel explicite avec la question. Ceux qui y parviennent le font en modifiant le texte, soit en ajoutant une phrase à P1, interrogeant Gabriel sur la raison de son émotion par une interrogation partielle : Pourquoi es-tu triste au lieu de Tu as perdu quelque chose?, ce qui permet d'enchainer la P2 j'ai perdu ma toupie chinoise (Dor CE2 PA V1V2); soit en substituant une question Qu'est-ce que tu cherches? à la P1 imposée, ce qui permet également l'insertion de la P2 (- Ma toupie chinoise).

7 élèves de fin de CM1 et de CM2 ajoutent une phrase de dialogue en amont :

Gabriel passe la porte de [l'école] et aperçois sa copine/ Anne-Lise dans la cour de récréations / - Salut Anne-Lise ! crie Gabriel en fouillant dans / ces poches / - Salut tu as perdu quelque chose? lui demande Anne-Lise/ - Ma toupie chinoise sur le chemin de l'école. (Chl CM1 PYR B V1)

11. Dans nos transcriptions, les barres obliques marquent le changement de ligne. Les graphies et la ponctuation des scripteurs sont reproduites d'après les copies. 
En CM1 et CM2 ce sont surtout des modifications plus profondes qui interviennent comme la transformation en interrogative indirecte ou le recours à du discours narrativisé.

(3)

Un jour Gabriel a perdu sa toupie sr le chemin/ de l'école/ Anne-Lise elle voyait regarder partout part terre./ Elle lui demanda ce qu'il avait perdu, Gabriel réponda : J'ai perdu ma toupie/ chinoise sur le chemin de l'ecole. (And CM2 PYR 2 V3)

6 élèves utilisent l'émotion de Gabriel pour lui en demander la cause. Pour ce faire ils utilisent d'abord un discours narrativisé et passent au discours direct avec la question imposée, parfois en la modifiant :

(4)

Il était une fois un petit garçon nomé Gabriel./ Il allait à l'école. Un jour en allant à l'école// il avait perdu sa toupie sans se rendre conte,/ est il arriva à l'école./ Il était boule verser, est une camarade de classe lui parla «:/ - Sa ne va pas? lui demenda Anne Lise/ Il lui réponda»:/ - Non j'ai perdu ma toupie!» (Wil CM1 PYR 2 V1)

Le passage de la généralité du DN à une raison précise au DD assure la cohérence du texte. Pour y parvenir, ces élèves recourent à l'utilisation d'inférences et à leur connaissance du monde comme certaines manifestations d'émotions (pleurs, bouderie, tristesse), qui peuvent faire comprendre que l'enfant est privé de l'objet qu'il convoite. Les élèves de CM2 qui utilisent ces inférences recourent plus rarement au dialogue alors que $5 \%$ des textes de CE2 sont entièrement dialogués, les phrases à insérer ayant transformé la totalité du texte en dialogue.

Le recours aux inférences s'accompagne de la quatrième règle de cohérence : la congruence. La question d'Anne-Lise est ainsi une conséquence des actions de Gabriel (il fouille, cherche autour de lui) et/ou de ses réactions (pleurs, tristesse, colère).

L'écriture de la deuxième version montre assez peu de modifications sur ces points.

7 élèves de CE2 et 5 élèves de CM2 de l'école la plus favorisée socialement ont cependant progressé en ce qui concerne les éléments permettant l'identification des locuteurs. Cette amélioration est liée aux demandes des enseignants, demande collective orale en CE2 précédant immédiatement la réécriture et demandes individuelles notées sur les copies concernées en CM2. Seule une enseignante de CM2 sur les 6 classes enquêtées permet aux élèves de retravailler l'insertion des phrases imposées. L'exemple suivant montre comment s'effectue 
la prise en compte des inférences chez une élève de CM2 en grande difficulté.

a) Un jour un jeune garçon avait perdu sa toupie chinoise est/ son amie lui demanda tu cherches quelque chose?/ Oui dit le jeune garçon. Je cherche ma toupie chinoise je les/ perdu.

b) V2

Anne Lise voie quil a perdu quel que chose parce qu'ils fouille / c'est poches les poches de son mento et il regarde partout.

c) V3 intègre le segment retravaillé en V2 :

Un jour un jeune garçon avait perdu sa toupie chinoise Anne lise/ voit qu'il a perdu quelque chose parce qu'ils fouille les poches de son/ manteau et il regarde partou./ Anne-lise lui demanda tu cherche quelque chose? Oui dit le jeune garçon. Je cherche ma toupie chinoise je l'ai perdu. (Bre CM2 PYR C V1)

\section{Les modifications syntaxiques : du texte à la phrase}

À l'inverse de ce que nous venons de voir, les contraintes sémantiques mais aussi linguistiques et syntaxiques du texte à produire ont des répercussions sur les phrases à insérer. Il semble que le plus souvent ces modifications laissent apparaitre par défaut les représentations que se font les scripteurs de la phrase.

\subsection{Segmentation et ponctuation}

Deux grandes lignes apparaissent à l'analyse.

- Majuscule et point.

23,7\% des copies de CE2 contre 16,8\% des copies CM2 ne mettent pas de majuscule aux phrases à insérer, que ce soit pour signaler le début du dialogue, ou le début de l'alinéa. En revanche, une petite minorité de productions de CE2 (moins de 10\%) utilise la majuscule pour le changement d'interlocuteur en l'absence d'autres marques. Mais la différence majeure vient surtout de l'absence de point clôturant traditionnellement la phrase graphique : $20,7 \%$ des copies de CE2 ne mettent pas de point avant la phrase précédant le dialogue, ni après l'incise, ni de point final au dialogue. Les auteurs de ces copies n'utilisent par ailleurs ni tirets ni guillemets, ni alinéas : c'est le changement de marques déictiques qui signale seulement la limite des phrases. Ce n'est plus le cas pour les CM2 : 8,9\% 
seulement des copies omettent le point à la fin du dialogue ou après la phrase d'incise.

- Marques de dialogue.

Entre le CE2 et le CM2 s'effectue un apprentissage formel. En effet $60,3 \%{ }^{12}$ des copies de CE2 ne font pas d'alinéa, et $61,3 \%$ n'utilisent pas les tirets pour détacher ces phrases de dialogue de la narration. Pour ces élèves, le texte apparait comme un bloc sans démarcation, entrainant parfois la confusion des plans : certains élèves rédigent tout le texte à la $1^{\text {re }}$ personne, ce qui peut être interprété comme une contamination du texte par les phrases de dialogue à insérer. Mais en CM2, il n'y a plus que $23,76 \%$ des copies sans alinéa et $26,7 \%$ sans tirets. En revanche, $44,5 \%$ des copies de CE2 et 10,8\% des copies de CM2 qui n'ont mis ni tiret ni alinéa utilisent les guillemets comme autre système pour marquer la rupture énonciative entre narration et dialogue.

\subsection{Segment introducteur et incise}

Deux modifications syntaxiques majeures sont à relever dans l'amont et l'aval de P1 et P2:

a) la substitution d'un segment introducteur à l'incise;

b) l'encadrement du DD par le cumul d'un verbe introducteur à gauche et de l'incise à droite (Boré, 2012).

Si les modifications apportées par a) sont pour la plupart destinées à répondre aux critères de cohérence (partie précédente), ce n'est pas le cas pour b) qui témoigne de difficultés syntaxiques persistantes.

- Pour a), quelques exemples de substitution d'un segment introducteur à l'incise montrent l'enchainement suivi :

(6)

Il était une foix un ga[r]çon du nom de Gabriel ${ }^{13}$ va à/ l'école avec sa toupie chinoise mais par accident il la perda. Une fois arriver dans l'école Anne-lise lui demande/ «-Tu as perdu quelque chose?» [...] (Em CE2 PA V1)

12. Nous n'avons pas détaillé les résultats sur la ponctuation au niveau du CM1 pour marquer le contraste entre CE2 et CM2.

13. Ici, procédé très fréquent qui consiste à mettre en «facteur commun» un nom de la phrase précédente servant de sujet à la phrase suivante sans (savoir?) utiliser le relatif. 
(7)

Gabriel enfant de douze ans court sur le chemin/ de l'école avec sa toupie chinoise préférée. Il tombe,/ pose sa toupie parterre pour voir la blessure et l'oublie! Pendant la récré, comme il veut y jouer/ avec sa meilleur amie (Anne Lise) il la cherche dans/ sa poche mais ne la trouve pas! Il s'exclame alors/ «oh non» Anne lise lui demande :/ - Tu as perdu quelque chose. [...] (Max CM1 PYRB V1)

(8)

Gabriel un petit garçon avait perdue ça toupie chinoise/ multicolores/ il était en train de la chercher, soudain une amie de gabriel lui demanda:/ - tu as perdu quelque chose [...] (Jas CM2 PYRC V1)

Les proportions décroissent avec les niveaux considérés : 24,5\% des copies (18 élèves) de CE2 substituent un verbe introducteur à l'incise, contre $18,2 \%$ des copies (10 élèves) pour le CM1 et 15,1\% pour le CM2 (9 élèves). Ces résultats sont difficiles à interpréter, car ce qui est parfois le signe d'une cohérence remarquable comme (7) peut aussi signaler une difficulté à utiliser l'incise : de fait, la plupart des élèves qui, dans leur version 1, utilisent un segment introducteur au lieu de l'incise, ajoutent cette dernière dans leur version 2 tout en conservant le segment introducteur et se retrouvent alors dans le cas b).

- Pour le cas b) (encadrement du DD), les résultats diffèrent totalement du cas a), en ne révélant aucune «progression» entre le CE2 et le CM2. Au contraire : on trouve moins de copies qui «encadrent» au CE2 (19,6\%) qu'au CM2 (22,7\%). La surprise vient du CM1 où l'on ne trouve d' «encadrement» que dans 7,3\% des copies avec la même proportion dans les deux CM1. Ces résultats doivent cependant être nuancés par la différence entre les deux versions. En effet, dans les trois niveaux, les scripteurs tiennent compte des remarques faites en V1 par les enseignants des six classes qui notent tous «répétition»sur les copies : c'est particulièrement vrai en CM2 où les «encadrements » représentaient $23,2 \%$ des copies en V1, mais n'étaient plus que $8 \%$ dans la V2. Le fait notable et constant réside cependant dans l'absence d'information véritablement «grammaticale» de la part des enseignants sur le fonctionnement syntaxique de l'incise. Soit l'exemple suivant «'encadrement» :

(9)

C'est l'histoire d'un petit garçon qui s'appelait/ Gabriel. Un matin il partit avec sa nouvelle/ toupie chinoise multicolore, il était très content de/ pouvoir l'emmener à l'école. Mais à un moment / il s'aperçut qu'il l'avait perdue, il était très/ déçu, triste, d'une humeur noire. Son amie 
Anne-/ lise lui demanda : / - tu as perdu quelque chose lui demandat-elle?/ - Ma toupie chinoise sur le chemin de l'école. (Em CM2 PYRC V1)

Nous avons choisi cet exemple car il émane d'une bonne copie, et que l'encadrement persistera dans la V2 de la scriptrice. Roubaud (2014) signale que l'encadrement se prolonge jusqu'en $6^{\mathrm{e}}$ voire en $5^{\mathrm{e}}$. L'incise n'est donc pas sentie comme une phrase autonome; elle est sans doute comprise comme une prolongation du DD, une formule figée dont la fonction semble beaucoup moins affirmée que celle du segment introducteur. On notera que les modifications par l'ajout d'un verbe introducteur + incise encadrant le DD touchent davantage les élèves de CM1/CM2 que ceux de CE2.

Pourtant des modifications significatives se produisent dans le texte avant l'incise, en CM2 où elle est «préparée» par un verbe de mouvement dans $7 \%$ des copies, qui proposent un segment introducteur de P1 préparatoire de l'incise mais différent du verbe de celle-ci utilisé en position finale :

C'est l'histoire de Gabriel. Il a perdu sa toupie chinoise/ en métal multicolore qui lance des note joyeuse. En allant à/ l'école il a laissé son sac ouvert avec sa toupie dedans, puis il/ s'est fait bousculé par un garçon qui courrait. Dans la cours / à l'école il s'est rendu compte que sa toupie multicolore avait/ disparue. Il était très malheureux et voulait même sortir pour/ aller la chercher. Mais son amie est venue :/

- Tu as perdu quelque chose? lui demanda Anne Lise/

- Oui ma toupie chinoise sur le chemin de l'école. (Naz CM2 JO V2)

Les phrases à insérer contraignent sémantiquement le texte qui à son tour les contraint syntaxiquement. En effet, l'incise présuppose souvent son locuteur connu du lecteur, alors que l'utilisation d'un segment introducteur précédant le DD (avec ou sans verbe de locution comme ici), permet généralement d'introduire aussi le locuteur du DD qui va suivre, avec la possibilité de le désigner de façon détaillée en amont de son discours. Cette différence n'est pas sans conséquence sur le fonctionnement de la nomination des locuteurs des phrases à insérer (notamment Anne-Lise, nouveau locuteur qu'il faut introduire dans l'histoire dont Gabriel est le héros premier nommé).

Dans l'exemple ci-dessus (10) l'incise n'est pas redondante: le verbe de mouvement du segment introducteur annonce l'actant, (information nouvelle) qui est alors nommé par l'incise, laquelle assume pleinement sa fonction de verbe de locution. 
Mais c'est beaucoup moins le cas pour l'exemple suivant (11), où verbe introducteur et verbe de l'incise, tous deux verbes de locution, sont redondants, brouillant la coréférence entre copine et Anne-Lise.

Un petit garçon de 9 ans només Gabriel a perdu sa toupie porte/ bonnheure. Il est très triste. Soudain sa copine lui dit :/ - Tu as perdue quelque chose? lui demanda Anne-Lise/ - Ma toupie chinoise sur le chemin de l'école. (ValP CE2 J0 V1)

\subsection{La phrase elliptique}

La caractéristique commune aux trois niveaux est le renforcement de la phrase averbale par la prophrase «oui», qui passe de 10,8\% en CE2 à environ un quart des copies en CM1 $(21,2 \%)$ et en CM2 (24,7\%). On note aussi conjointement :

- l'ajout d'un verbe dans la phrase elliptique (averbale). Pour tous les scripteurs qui le font, cet ajout reprend le verbe de P1, avec changement des marques de la deixis en «j'ai perdu», cumulé avec la prophrase «oui». Ce fait se produit dès la V1 et demeure inchangé en V2;

- l'ajout d'autres phrases dans la phrase elliptique : très peu de copies en CE2 (2,8\%), et en CM1 (5\%) ajoutent d'autres phrases que celles du texte. La rupture se produit au CM2 où 10,8\% des copies y ont recours, la plupart du temps pour en expliciter le contenu. On observe ainsi un développement continu de P2, soit par l'ajout de propositions $(6,9 \%)$ soit par l'ajout d'un verbe introducteur $(12,8 \%)$ en concurrence avec une incise $(11,8 \%)$;

- l'ajout d'une incise précisant l'allocutaire, symétrique de la question P1. C'est le cas pour 7,9\% des copies de CE2, pour $25 \%$ de CM1 (mais $11,25 \%$ de copies utilisent un segment introducteur) et pour 11,8\% en CM2 (avec 12,8\% de segments introducteurs.

Ces modifications dans P2 nous semblent indiquer chez les scripteurs une prise en compte des particularités énonciatives de la phrase elliptique. En effet, si nous suivons Kuyumkuyan (2013, p. 145), les réponses elliptiques de ce type ne sont pas

[...] une reconduction pure et simple des propos de l'interlocuteur.

Ce qu'a dit ce dernier n'est pas ce sur quoi enchaîne le locuteur. La réplique réactive ne s'appuie pas sur une reproduction à l'identique de 
la précédente intervention, mais sur sa représentation par le locuteur, représentation implicitée et toujours plus ou moins fidèle comme le rappelle O. Ducrot (1984:199).

Nous le vérifions avec les phrases de ce dialogue imposé. Ainsi, à la question du locuteur (Anne-Lise) :

Tu as perdu quelque chose?

la réponse reconstituée serait :

[J'ai bien perdu [quelque chose]] ma toupie chinoise...

avec changement des marques de la deixis et une fonction du GN ma toupie chinoise, plutôt apposition que COD du verbe ellipsé. Nous suggérons qu'il ne s'agit pas ici d'une coénonciation formant un tout, mais de deux énonciations différentes, les deux locuteurs étant distingués par des marques différentes d'énonciation, et produisant chacun un acte illocutoire distinct. C'est précisément ce que tente de faire un petit nombre d'élèves qui semblent traiter un système nécessitant de leur part une identification du rôle des locuteurs au travers de phrases de niveau (incise vs système interrogatif comportant une phrase ellipsée) et de fonction (question-réponse avec changement de la deixis) distincts.

\section{Conclusion}

Nous avons donc tenté, à partir d'une consigne commune d'insertion de phrases imposées dans un corpus de productions écrites de cycle 3 , d'observer les relations entre celles-ci et le texte à produire.

La détermination de ces phrases de dialogue sur le texte s'exerce principalement par les contraintes sémantiques, qui pèsent fortement sur le cotexte amont. Dans les réponses apportées à ces contraintes, c'est au niveau du CM2 que s'observe la rupture, les niveaux précédents juxtaposant leur texte avec les phrases à insérer sans modification substantielle de l'un par l'[es]autre[s]. Les réponses en CM2 se traduisent d'abord syntaxiquement par une représentation plus claire de plans d'énonciation distincts entre le texte amont et le dialogue, ce qui se marque par une plus grande régularité dans la segmentation en paragraphes, une ponctuation spécifique et le changement de la deixis entre les deux plans. Mais, plus profondément, c'est la prise en compte de l'implicite dont sont porteuses ces phrases qui les conduit à en modifier 
la syntaxe par l'ajout d'éléments explicatifs entrant dans la structure des phrases à insérer : c'est ainsi que les élèves sont conduits à modifier la structure interrogative en question plus large ou au contraire plus partielle et à transformer la phrase averbale-elliptique en phrase canonique, voire à la développer, mettant au jour les éléments énonciatifs dont elle est porteuse. Les résultats sont paradoxaux, car, en faisant de telles modifications, les élèves rencontrent des difficultés inédites, telles que l'incompatibilité entre segment introducteur et incise et la gestion des chaines anaphoriques. Paradoxalement, ils montrent aussi qu'ils ont intériorisé une conception scolaire-académique de la phrase.

Sur le plan didactique, il apparait que les maitres sont très peu conscients de la nécessité de traiter les phrases dans et avec le texte : les remarques restent ponctuelles et font l'impasse sur l'analyse de l'insertion de ces phrases de dialogue, dont on rappelle qu'elles sont issues d'un texte écrit extrait d'un album, et non pas créées artificiellement pour un exercice. Les rappels magistraux concernent surtout la présentation et la ponctuation du dialogue et sont excessivement formels, à l'exception d'une classe où l'enseignante engage un travail en profondeur dans la réécriture. Ce constat devrait se traduire par une sensibilisation accrue à l'analyse textuelle des copies dans la formation des maitres, générant la conviction que le sens prime la signification, ce qui modifie la posture énonciative du scripteur à l'origine du propos à tenir, en affectant solidairement le texte et la phrase.

\section{RÉFÉRENCES BIBLIOGRAPHIQUES}

Authier-Revuz, Jacqueline. (1995). Ces mots qui ne vont pas de soi: boucles réflexives et non-coüncidences du dire (tomes I et II; $1^{\text {re éd.). }}$ Paris : Larousse.

Benveniste, Émile. (1966). Problèmes de linguistique générale (tome 1, chap. $x$ «Les niveaux de l'analyse linguistique»). Paris : NRF, Gallimard ( $1^{\text {re }}$ éd. 1964).

Boré, Catherine. (2012). Le discours direct dans des écrits fictionnels scolaires : marques et signification. Dans S. Branca-Rosoff, C. Doquet, J. Lefebvre, É. Oppermann-Marsaux, S. Pétillon \& F. Sitri (dir.), L'hétérogène à l'œuvre dans la langue et les discours. Hommage à Jacqueline Authier-Revuz (p. 117-134). Limoges : Lambert-Lucas.

Charolles, Michel. (1978). Introduction aux problèmes de la cohérence des textes: Approche théorique et étude des pratiques pédagogiques. Langue française, 38, 7-41. 
Charolles, Michel. (2005). Analyse de discours, grammaire de texte et approche grammaticale des faits de textualité. Le français aujourd'hui, 148, 33-45.

Combettes, Bernard \& Charolles, Michel. (1999). Contribution pour une histoire récente de l'analyse du discours. Langue française, 121, 76-116.

Danlos, Laurence, SAgot, Benoît \& STERn, Laurence. (2010). Analyse discursive des incises de citation. Dans F. Neveu, V. Muni Toke, J. Durand, T. Klingler, L. Mondada \& S. Prévost (dir.), Actes du Congrès mondial de linguistique française (p. 2237-2254). EDP Sciences. <http://dx.doi. org/10.1051/cmlf/2010217>.

Deloor, Sandrine. (2012). Bref aperçu historique des travaux sur la présupposition. Langages, 186, 3-20.

Kuyumkuyan, Annie. (2013). Les séquences fragmentaires en contexte dialogal : l'interface syntaxe/énonciation. Dans P. Hadermann, M. Pierrard, A. Roig \& D. Van Raemdonck (dir.), Ellipse et fragment : morceaux choisis (p. 143-156). Bruxelles : Peter Lang.

LACHERET-Dujour, Anne et coll. (2011). Oui mais elle est où la coupure, là? Quand syntaxe et prosodie s'entraident ou se complètent. Langue française, 170, 61-79.

LEFEUVRE, Françoise. (2013). Les réponses aux questions : énoncés fragmentaires, elliptiques ou bien autonomes? Dans P. Hadermann, M. Pierrard, A. Roig \& D. Van Raemdonck (dir.), Ellipse et fragment : morceaux choisis (p. 81-97). Bruxelles : Peter Lang.

Plane, Sylvie. (2006). Singularités et constantes de la production d'écrit. L'écriture comme traitement de contraintes. Dans J. Laffont-Terranova \& D. Colin (dir.), Didactique de l'écrit. La construction des savoirs et le sujet-écrivant (p. 33-54). Namur : Presses universitaires de Namur.

RAstier, François. (2005). Mésosémantique et Syntaxe. Texto! Textes \& Cultures.<www.revue-texto.net/Inedits/Rastier/Rastier_Mesosemantique.html> (consulté le 10 novembre 2013).

Riegel, Martin, Pellat, Jean-Christophe \& Rioul, René. (1994). Grammaire méthodique du français ( $1^{\text {re }}$ éd.). Paris : Presses universitaires de France.

Roubaud, Marie-Noëlle. (2014). De la description de la langue à son enseignement (Habilitation à diriger des recherches, non publiée). Université Stendhal-Grenoble 3, Grenoble. 